\title{
The Demographic Effect of "Lucky" Breeding: Consequences of a Single Exceptional Breeding Result
}

\author{
By \\ Mircea Pfleiderer and Jörg Pfleiderer \\ (vorgelegt in der Sitzung der math.-nat. Klasse am 17. Juni 2010 durch \\ das w. M. Jörg Pfleiderer)
}

\begin{abstract}
We combine two aspects of fitness - lifetime reproductive success and (population) growth rate ("propensity fitness") - for defining a generation duration which, in combination with either of these fitness definitions, can quantitatively answer one of the classic questions of evolution: What is the effect, on later generations, of a single case of reproduction greater (or smaller) than average? Individual breeding success of offspring can be included by a simple multiplication of generations, or by referring the reproductive history directly to grandchildren or later generations. We define inclusive fitness by an inclusive lifetime reproductive history, combining the histories of two individuals sharing an altruistic act. HAMILTON's rule should rather be expressed as a ratio of inclusive growth rate fitnesses. The termination of growth/decline after a few generations or within one generation, respectively, has different results so it is mandatory to distinguish clearly between these two cases of constant vs. waxing and waning populations. Observed life reproductive histories need a reduction to successful histories (i.e., reproductive offspring) for quantitative answers.
\end{abstract}

Key words: Individual fitness, inclusive fitness, lifetime reproductive history, reproductive success, generation length, HAMILTON's rule.

\section{Introduction}

Individual fitness is supposed to be a measure of the reproductive success of an individual as translated into future generations. Origin- 
ally, it was essentially the number $S$ of offspring produced in a lifetime (lifetime reproductive success LRS) that was used as fitness measure (often, but not always, as a ratio to a maximum LRS). It soon became clear that the time of reproduction and thus the life history of reproduction play a major role. This is where the concept of fitness as a (population) growth rate (propensity fitness) was introduced. MCGRAW and CASWELL ([5], henceforth MG\&C) considered a "population projection matrix" the positive eigenvalue $\lambda$ of which is an "individual growth rate" (or "asymptotic growth rate") that is a measure of the exponential population development introduced by a given reproductive success.

The primary information in both cases is the lifetime reproductive history (LRH). MG\&C give, as example, the history of a particular bird (blue tit, Parus caeruleus, individual 1493900) as (3.5, 5, 5, 6, 4.5) which means that the bird produced 3.5 offspring "of its own genotype" in the first year (i.e., 7 together with the partner), 5 (10) offspring in the second and third, 6(12) in the fourth, 4.5 (9) in the fifth year and stopped reproduction thereafter. The corresponding fitness numbers are $S=24$ (LRS) and $\lambda=4.82$ (growth rate per year). To our knowledge, there is no other fitness definition derived directly from the LRH.

BROMMER et al. [1] have discussed empirical uses of either definition. Their main result is, not surprisingly, that both forms have their advantages. Which form seems to give more reliable results depends on the question asked.

Indeed, the definition of fitness as total lifetime reproductive success can give insight into problems where the modern growth rate definition is of little use. Probably the oldest example goes back to MALTHUS and DARWIN: the population explosion following from an average LRS greater than 1 per individual, regardless of the generation length, or of when the offspring is raised (i.e., the propensity fitness is - nearly irrelevant). For stable conditions, some offspring have to die without reproducing - let us call this the natural death toll or non-reproduction toll. LEYHAUSEN [4] tried repeatedly to convey this simple but important fact, which scientists often take too much for granted to be even considered, to laymen who need this information in order to make decisions on environmental politics.

Note that it is the average LRS that is restricted, not the individual LRS as an exception. The difference between average and exceptional fitness is, in many publications, not very clearly mentioned, perhaps because there seems to be a tendency to consider any individual fitness as of mainly hereditary origin. 
Of course, there are cases in which the population does indeed explode, for example when filling an empty niche, but then the explosion must necessarily - and indeed does - stop after a while. Also, it is generally preceded by a corresponding population implosion that was necessary for creating the niche.

On the other hand, the growth rate definition of fitness gives insight into other problems where the older total-reproduction definition fails. For example, the time needed to fill a niche (and, by that, the ability to fill a niche) essentially depends only on the possible growth rate. A fly with a multiplying time of weeks is so superior over a predating beetle with a one-year cycle that it is useless to keep applying fly poison that also kills the beetles but closes the niche to the flies for only a short time [3]. Here, the number of offspring is (nearly) irrelevant. As before, the argument rests on the average fitness, not at all on an individual and exceptional one.

A problem that needs both definitions to be understood is the following: What is the best average strategy for a species living in niches that frequently open (and, of course, close again, killing the current population). Apparently, a high growth rate is useful. This is accomplished for any genotype as well by, say, a twofold one-time reproduction (4 per pair) in a given time as by a fourfold one-time reproduction ( 8 per pair) in twice the time. But in average conditions (no open niche, constant population) the first case means that $1 / 2$ of the offspring ( 2 of 4 ) must die without reproducing while the second case requires a higher toll of $3 / 4$ (viz., 6 of 8 ). That is to say, a small generation length has advantages, which means a smaller total reproduction (fitness definition one) with given growth rate (fitness definition two), as well as a larger growth rate with given total reproduction.

The evolutionary answer to these problems is well-known (e.g., [6]). The $r$-strategy (for niche filling) prefers short generations and many offspring while the $K$-strategy (for essentially constant population) puts emphasis on long generations, few offspring and parental care.

\section{Generation Length and Its Application to Individual Fitness}

The foregoing examples use fitness in the same sense in which it seems most often to be understood, viz., as a property or average property of species, populations, sub-populations, or types within populations. Such fitness is always meant to be valid over many 
generations, even if this is hardly ever stated. There is no need to distinguish between generations, and thus no need to follow the course of overlapping generations. For example, if all animals had the same (average) growth rate fitness $\lambda$, then the total population would grow incrementally with $\lambda$. Or, if an individual had fitness $\lambda$ and its entire offspring inherited properties giving rise to the same (average) fitness, then the descendants of this individual would increase asymptotically (i.e., after a few generations) with this same growth rate, $\lambda$. It is of no interest how many generations a certain development needs.

Observed individual fitnesses are, in most cases, used as probes in a statistical sample, that is, used as an indication of how large the average fitness might be, especially in qualitative comparison with another observed fitness of an individual showing other properties or other traits. In other words, they are used as an indication of how different properties or behaviour might influence the average fitness and thus be of evolutionary consequence.

However, things become different if we want to consider a single individual and the singular effect of its individual fitness: the impact of the "lucky" (or, as well, "unlucky") breeder. Even the simplest case of reproduction, i.e., only once a lifetime, contains two items of information: the number of offspring and the age at reproduction, i.e. the generation length. It is not possible to describe those two items by one number only. In general, it is the lifetime reproductive history LRH that gives the full amount (or, at least, a fuller amount) of information. Thus, even two numbers cannot do more than approximate to what is really happening.

In the LRS definition of fitness, one misses information about when the offspring is born, or at least information on the average generation length. In the growth rate definition, information is missing about how long the growth rate is valid. One could speculate on a growth rate that changes according to offspring already born. A simpler approach would be to estimate an average generation length as the time of validity for the original growth rate.

Neither fitness definition alone can quantitatively answer the classic question of fitness theory for which the concept of individual fitness was originally invented: to be a measure of the reproductive success of one single individual as translated into future generations. But a combination of both can, and each can separately if combined with a generation length that is determined from both fitness numbers. We define a medium or effective generation length $T$ by the time it takes an exponential growth with rate $\lambda$ (as 
following from the population projection matrix) to produce the total offspring $S$, that is,

$$
T=\ln S / \ln \lambda .
$$

The lifetime reproductive history is thus approximated by a onetime reproduction, at age $T$, of the total offspring $S$, or, alternatively, by a continuous exponential increase (or decrease) with $\lambda$ for time $T$. The latter interpretation shows that the name "individual growth rate" for $\lambda$ was indeed well-chosen. Instead of following up the detailed life histories for generations that partly overlap, one can follow up the consecutive row of average generations.

The answer to the question what the effect of a single case of reproductive success greater (or smaller) than average would be on later generations is quite simple but it is, of course, an approximation. The three "fitness numbers" $S, \lambda$, and $T$ of which only two are independent replace the fuller information of the LRH, which itself is an approximation. Let the average LRH yield fitness numbers $S_{0}, \lambda_{0}, T_{0}$ while the exceptional breeder - "exceptional" meaning primarily that it is a single case, the offspring and later generations being as average as all others has fitness numbers $S, \lambda, T$. Then the latter will breed with $\lambda$ for time $T$ while the average animal breeds, during the same time, with $\lambda_{0}$, or, alternatively, the latter will produce its offspring $S$ in time $T$ while the average animal will, in the same time $T$, encounter $T / T_{0}$ generations and produce a total offspring $S_{0} * *\left(T / T_{0}\right)$, where the double asterisk means "to the power of" and stands for an exponential with easier readability of indexed exponents. Thus the ratio $x$ of offspring of the exceptional breeder and of the average breeder will later be

$$
x=\left(\lambda / \lambda_{0}\right)^{T}=\left(\lambda / \lambda_{0}\right) * * T=S /\left(S_{0} * *\left(T / T_{0}\right)\right) .
$$

This simple recipe, which we illustrate below by examples, shows clearly that the problems for which the concept of fitness was originally invented need more than one number to be quantitatively answered. The growth rate alone is sufficient to distinguish between lucky $(x>1$, $\left.\lambda>\lambda_{0}\right)$ and unlucky $\left(x<1, \lambda<\lambda_{0}\right)$ breeder but the generation length of the exceptional breeder is needed for quantification.

The examples include the case of one single reproductive success ("lucky/unlucky breeder"); the model of unusual reproductive success in more than one generation (multiplication of generations); the effects of the necessary termination of any unlimited growth (waxing and waning $v s$. constant population); and finally the question of inclusive fitness (altruistic acts, where at least two individuals - donor and 
recipient - with different reproduction details and different direct fitness have to be combined).

\section{Examples of Lucky Breeding}

We now show how the combination of either fitness number, $S$ or $\lambda$, with the generation length $T$ may be used. The examples are chosen to be quite simple in order that the reader may check the calculations without much effort. Most examples deal with growing populations, the simple reason being that one can work with integers, which makes things easier without limiting the generality at all. For the calculation of the growth rate we use, as pendant to the individual lifetime reproductive success $S$, the individual form of the matrix that yields the characteristic equation $\Sigma s_{i} / \lambda^{-i}=1$, where $\left(s_{1}, s_{2}, \ldots\right)$ is the life reproductive history (see MG\&C).

In the first example, we ask what influence one individual with increased fitness number of either definition - this is the lucky breeder has on the further development of the population with the restriction that the offspring does not apply the same increased reproduction but rather behaves normally, i.e., shows average reproduction: the luck does not continue. The "lucky mate" or the "unlucky breeder" (reduced reproduction) could be treated similarly.

We assume a species or population that reproduces with an average of 1 each per parent (i.e., 1 with probably the same genomic type as the parent in question, or 2 offspring per pair) in each of the first and second years (or time unit). The (average) LRH is thus $(1,1)$ or, as well, $(1,1,0)$ no offspring in the third year -, with $S_{0}=2, \lambda_{0}=1.62, T_{0}=1.44$. Let one individual, the "lucky breeder", have a different LRH of $(1,1,1)$, i.e., it adds another 1 offspring ( 2 per pair) in its third year, giving $S_{1}=3$, $\lambda_{1}=1.84, T_{1}=1.80$.

Let us first consider the further development in detail (see Table 1). Table 1 should be understood as giving, except for the original parents, average numbers in at least two respects: an average concerning the LRH as well as an average concerning the probability that an offspring has the same genomic type as the individual parent. We give definite numbers instead of probabilities in order to be able to compare them with our estimates $x$. The counts are given for ten years to show the gradual development towards an exponential increase.

In the first year, 1 offspring with the same type as the parent individual is born (this is an average). In the second year, 1 offspring of the parent (second year) as well as 1 grandchild (first-year offspring 
Table 1. Birth development for 10 years (examples 1 and 2). (a) normal case, (b) one lucky breeder, (c) hereditary well-breeder (hereditary increased reproductive success), (d) average continuation of a lucky breeder with one lucky child into the second generation (see text). Total number born in a year (bold) and their offspring in following years (fine) according to the LRH. Ratio of births to last year (italic)

\begin{tabular}{|c|c|c|c|c|c|c|c|c|c|c|c|}
\hline Year & 0 & 1 & 2 & 3 & 4 & 5 & 6 & 7 & 8 & 9 & 10 \\
\hline $\begin{array}{l}\text { normal } \\
\text { breeder } \\
\text { LRH }=(1,1)\end{array}$ & $\begin{array}{l}1 \\
-\end{array}$ & $\begin{array}{l}1 \\
\mathbf{1} \\
1.0\end{array}$ & $\begin{array}{l}1 \\
1 \\
2 \\
2.0\end{array}$ & $\begin{array}{l}1 \\
2 \\
\mathbf{3} \\
1.5 \\
\end{array}$ & $\begin{array}{l}2 \\
3 \\
5 \\
\end{array} 67$ & $\begin{array}{l}3 \\
5 \\
8\end{array}$ & $\begin{array}{l}5 \\
8\end{array}$ & 8 & & & \\
\hline $\begin{array}{l}\text { lucky } \\
\text { breeder } \\
\text { LRH }=(1,1,1) \\
\text { then }(1,1)\end{array}$ & $\begin{array}{l}1 \\
-\end{array}$ & $\begin{array}{l}1 \\
\mathbf{1} \\
1.0\end{array}$ & $\begin{array}{l}1 \\
1 \\
2 \\
2.0\end{array}$ & $\begin{array}{l}1 \\
1 \\
2 \\
4 \\
2.0\end{array}$ & $\begin{array}{l}2 \\
4 \\
6 \\
1.5 \\
\end{array}$ & $\begin{array}{l}1.60 \\
\\
4 \\
6 \\
\mathbf{1 0} \\
1.67\end{array}$ & $\begin{array}{l}13 \\
1.63 \\
\\
6 \\
10 \\
16\end{array}$ & $\begin{array}{l}13 \\
21 \\
1.62 \\
\\
10 \\
16\end{array}$ & $\begin{array}{l}13 \\
21 \\
\mathbf{3 4} \\
1.62 \\
\\
16\end{array}$ & $\begin{array}{l}21 \\
34 \\
\mathbf{5 5} \\
1.62\end{array}$ & $\begin{array}{l}34 \\
55 \\
89 \\
1.62\end{array}$ \\
\hline $\begin{array}{l}\text { hereditary } \\
\text { well-breeder } \\
\text { LRH }=(1,1,1)\end{array}$ & $\begin{array}{l}1 \\
-\end{array}$ & $\begin{array}{l}1 \\
\mathbf{1} \\
1.0\end{array}$ & $\begin{array}{l}1 \\
1 \\
2 \\
2.0\end{array}$ & $\begin{array}{l}1 \\
1 \\
2 \\
4 \\
2.0\end{array}$ & $\begin{array}{l}1 \\
2 \\
4 \\
7 \\
1.75\end{array}$ & $\begin{array}{l}2 \\
4 \\
7 \\
13 \\
185\end{array}$ & $\begin{array}{l}4 \\
7 \\
13 \\
\mathbf{2 4}\end{array}$ & $\begin{array}{l}\mathbf{2 6} \\
1.63\end{array}$ & $\begin{array}{l}26 \\
42 \\
1.62\end{array}$ & $\begin{array}{l}26 \\
42 \\
\mathbf{6 8} \\
1.62\end{array}$ & $\begin{array}{l}42 \\
68 \\
\mathbf{1 1 0} \\
1.62\end{array}$ \\
\hline $\begin{array}{l}\text { generation } \\
\text { addition } \\
\text { (average of } \\
\text { one of three) } \\
\text { LRH }=(1,1,1) \\
\text { then }(1,1,1 / 3)\end{array}$ & $\begin{array}{l}1 \\
-\end{array}$ & $\begin{array}{l}1 \\
\mathbf{1} \\
1.0\end{array}$ & $\begin{array}{l}1 \\
1 \\
\mathbf{1} \\
\mathbf{1} \\
2.0\end{array}$ & $\begin{array}{l}1 \\
1 \\
1 \\
1 \\
\mathbf{1} \\
\mathbf{3} \\
2.0\end{array}$ & $\begin{array}{l}0.33 \\
1 \\
1 \\
1 \\
3 \\
\mathbf{6 . 3 3} \\
1.58\end{array}$ & $\begin{array}{l}0.33 \\
1 \\
3 \\
6.33 \\
\mathbf{1 0 . 6 6} \\
1.68\end{array}$ & $\begin{array}{l}0.33 \\
6.33 \\
10.66 \\
\mathbf{1 7 . 3 3} \\
1.63\end{array}$ & $\begin{array}{l}10.66 \\
17.33 \\
\mathbf{2 8} \\
1.62\end{array}$ & $\begin{array}{l}17.33 \\
28 \\
\mathbf{4 5 . 3 3} \\
1.62\end{array}$ & $\begin{array}{l}28 \\
45.33 \\
\mathbf{7 3 . 3 3} \\
1.62\end{array}$ & $\begin{array}{l}44 \\
81 \\
149 \\
\mathbf{2 7 4} \\
1.84 \\
\\
\\
\\
\\
45.33 \\
73.33 \\
\mathbf{1 1 8 . 6 6} \\
1.62\end{array}$ \\
\hline
\end{tabular}

of the first-year child) of the same type is born on average, making together 2 of the same type born in that year. All of the offspring is assumed to reproduce normally with $(1,1)$. Following this further in the first 10 years, the average number of offspring born each year and having the same type as the original parent is 1, 2, 3, 5, 8, 13, 21, 34, 55, 
and finally $B_{0}=89$ (where $B$ is the number of births after 10 years) if the parent has the usual or average LRH $(1,1)$. The increased reproductive success of the lucky breeder with $\operatorname{LRH}(1,1,1)$ renders, instead, 1, 2, 4, $6,10,16,26,42,68, B_{1}=110$ if all offspring is assumed to have an average LRH of $(1,1)$ and not the increased one (the "luck" does not continue). Both rows of numbers increase, after the first years, with the "normal" growth factor of $\lambda_{0}=1.62$, but the fraction $x$ of specimens of the genomic type of the "successful" individual has increased, as compared to the offspring of one "normal" individual with another (its own) genomic type, by $B_{1} / B_{0}=110 / 89=1.24$, or $24 \%$, and this holds for all further generations.

Considering the same problem with only the fitness numbers given, the simplest approach would be that the "different" individual reproduces at its own growth rate $\lambda_{1}=1.84$ for one generation length $T_{1}(1.80$ years or time units) compared with a "normal" individual reproducing at its reduced growth rate $\lambda_{0}=1.62$ for the same time, while later on all offspring reproduce with the same, normal growth rate of 1.62 . The result, $x=\left(\lambda_{1} / \lambda_{0}\right) * * T_{1}=(1.84 / 1.62)^{1.80}=1.26$ or $26 \%$ is sufficiently close to the true result which uses the complete reproduction information instead of only the two fitness numbers.

Another interpretation of the same formula is as follows: In the generation time $T_{1}=1.80$ in which the "different" individual produces its offspring $S_{1}=3$, the normal individuals with $T_{0}=1.44$ already enter the next generation and thus produce more than the one-generation offspring $S_{0}$. The corresponding estimate with $S$ and $T$ is $x=$ $S_{1} /\left(S_{0} * *\left(T_{1} / T_{0}\right)\right)=3 /(2 * * 1.25)=1.26$.

The reason for the slight discrepancy between the calculated factor 1.26 and the true factor 1.24 can be found in an effect aptly described by the second well-chosen name for $\lambda$ as used by MG\&C, viz., "asymptotic growth rate". The growth rate of the offspring is reached only after a few generations (cp. Table 1). That is the same as assuming that the growth rate starts working at a time $t$ that is not zero. For instance, the "normal" outcome after 10 years is not $\lambda_{0} * * 10=124$ but rather $B_{0}=89=\lambda_{0} * * 9.3$, implying $t_{0}=10-9.3=0.7$. The corresponding number for the enhanced growth rate 1.84 of $\mathrm{LRH}(1,1,1)$ over 10 years would give (Table 1) $B^{*}=274=1.84 * * 9.2$, or $t=0.8$. Using this information (which goes beyond our 2-number approximation), a better estimate for the increase factor would be $x=\left(\lambda_{1} /\right.$ $\left.\lambda_{0}\right) * *\left(T_{1}+t_{0}-t\right)=1.136 * * 1.7=1.24$.

The second example shows that things become only slightly more complicated if an LRH is heritable but not inherited or not displayed by the entire offspring, or if some of the offspring show again, by good 
or bad luck, LRHs that differ from the normal case. We again take the "successful" individual of the first example, with LRH $(1,1,1)$. If all 3 first-generation offspring had the increased LRH but any of the following generations did not, then we would expect the increased growth rate to last for two generations, yielding $x=$ $\left(\lambda_{1} / \lambda_{0}\right) * * 2 T_{1}=1.59$, close to the direct result of 1.53 (i.e., calculated according to the method of Table 1) but larger due to the abovementioned asymptotic effect.

Assume now that only one of the offspring displays the same increased LRH, while the other 2 as well as all grandchildren and further generations (including the children of the "successful" child) behave normally, with history $(1,1)$. If it is the first (second, third) child that is "successful", the total ten-year outcome (number of births after 10 years) is $123(118,115)$, or a factor of $1.38(1.33,1.29)$ above the "normal" result of 89. The average (Table 1 ) is 119 , a factor of 1.34. Our two-number approximation can, of course, only reproduce the effect of an average child. It is born after 1 generation length, $T_{1}=1.8$, and starts reproduction correspondingly later. Its average LRH is $(1,1,1 / 3)$ with $S_{2}=2.33, \lambda_{2}=1.70, T_{2}=1.60$. Multiplication of generations gives $\left.x=\left(\left(\lambda_{1} / \lambda_{0}\right) * * T_{1}\right) *\left(\left(\lambda_{2} / \lambda_{0}\right) * * T_{2}\right)\right)=1.36$.

Alternatively, the two generations can be combined into a double generation for which the grandchildren are counted instead of the children. This gives an LRH of $(0,1,2,2.33,1.33,0.33)$ with $S=7$, $\lambda=1.77, T=3.40, x=\left(\lambda / \lambda_{0}\right) * * T=1.35$.

Playing around a little more with generation multiplication, consider the case of a lucky breeder $(1,1,1)$ whose 3 children are normal $(1,1)$ but all the 6 grandchildren are again lucky $(1,1,1)$, the great-grandchildren normal, their children again lucky and so on. The direct count according to the scheme of Table 1 (but not included there) gives 174 births in the tenth year, or an 174/89 $=1.96$-fold increase. The ten years correspond very nearly to 3.0 double-generations with a grandchildren-LRH $=(0,1,2,2,1), S=6, \lambda=1.74, T=3.24$, yielding a gain of $\left(\lambda / \lambda_{0}\right) * *(3 * T)=2.00$, or just to 3 lucky plus 3 normal generations yielding $\left(\lambda_{1} / \lambda_{0}\right) * *\left(3 * T_{1}\right)=2.00$.

These examples dealt with growing populations. No population can grow forever. Discontinued growth, or a reduction to the original level, will not change our results if started only after a few generations.

Take, for instance, the first example. There are three categories of animals, viz., no longer reproducing (more than two years old), reproducing once more (between one and two years old), and reproducing twice more (youngsters, less than one year old). Comparing the 
two cases "normal" and "successful", all of these three categories are present in one and the same ratio, 1.24. That is, whatever way we choose to reduce the population, whether suddenly or more slowly, whether more the old or more the young population, that ratio will remain, unless the reduction was not neutral, i.e., was able to affect the offspring of the two cases differently.

The situation changes, however, if we consider discontinued growth or reduction to constant population within one generation. We illustrate this by another version of the first example.

Constant population requires average fitnesses $S=1, \lambda=1$ (projection matrix of the population), which means in our first example that one-half of the total offspring $S_{0}=2$ will finally not reproduce and should thus not be integrated into the LRH. The "normal" (average) history $(1 / 2,1 / 2)$ gives $S_{9}=1, \lambda_{9}=1$ (individual projection matrix), $T_{9}=1.5$ which levels off, after a few years, towards $2 / 3$ births per year (1.0 births per generation length $T_{9}=1.5$ years). The lucky breeder, also losing $1 / 2$ of its offspring, has history $(1 / 2,1 / 2,1 / 2)$ and $S_{10}=1.5$, $\lambda_{10}=1.24, T_{10}=1.88$. If, again, all descendants have the normal average history $(1 / 2,1 / 2)$, this levels off towards 1.0 births per year, up by a factor of 1.5. Indeed, $\left(\lambda_{10} / \lambda_{9}\right) * * T_{10}=S_{10}=1.5$. Table 2 illustrates

Table 2. Early and late births. N: Normal breeder; all later generations are assumed normal

\begin{tabular}{lllll}
\hline LRH & $S$ & $\lambda$ & $T$ & $x$ \\
\hline 1,1 & 2 & 1.62 & 1.44 & $\mathrm{~N}$ (waxing) $=1.0$ \\
2,0 & 2 & 2.00 & 1.00 & 1.23 \\
0,2 & 2 & 1.41 & 2.00 & 0.76 \\
2,1 & 3 & 2.41 & 1.25 & 1.54 \\
1,2 & 3 & 2.00 & 1.59 & 1.40 \\
$1,1,1$ & 3 & 1.84 & 1.80 & 1.26 \\
$0.5,0.5$ & 1 & 1.00 & 1.50 & $\mathrm{~N}$ (constant) $=1.0$ \\
1,0 & 1 & 1.00 & 1.00 & 1.00 \\
0,1 & 1 & 1.00 & 2.00 & 1.00 \\
$1,0.5$ & 1.5 & 1.37 & 1.30 & 1.50 \\
$0.5,1$ & 1.5 & 1.28 & 1.64 & 1.50 \\
$0.5,0.5,0.5$ & 1.5 & 1.24 & 1.88 & 1.50 \\
$0.25,0.25$ & 0.5 & 0.64 & 1.56 & $\mathrm{~N}$ (waning) $=1.0$ \\
$0.5,0$ & 0.5 & 0.50 & 1.00 & 0.78 \\
$0,0.5$ & 0.5 & 0.71 & 2.00 & 1.23 \\
$0.5,0.25$ & 0.75 & 0.81 & 1.36 & 1.38 \\
$0.25,0.5$ & 0.75 & 0.84 & 1.70 & 1.60 \\
$0.25,0.25,0.25$ & 0.75 & 0.87 & 2.06 & 1.88 \\
\hline
\end{tabular}


quantitatively the well known fact ( $r$ - and $K$-strategic reproduction) that time of birth plays a role in waxing and waning populations but not in a constant one.

That is to say, it is useful to distinguish clearly between more or less unlimited growth for several generations (niche filling) with later cessation of growth (full niche) or reduction (niche emptying) on the one hand and, on the other hand, essentially-zero growth of levelled populations living in equilibrium with the environment. Observed LRHs will often be a mixture of those two cases, part of the offspring not reproducing again (when it should be removed from the LRH for relevant fitness numbers), the other part inducing growth for several generations followed by a later decrease (or vice versa). What is needed for quantitative answers is not an observed LRH but a successful LRH.

\section{Altruistic Acts and Inclusive Fitness}

Here, we deal with the effect of one single altruistic act on later generations. To our knowledge, no valid definition of inclusive growth rate fitness (inclusive propensity fitness) has hitherto been put forward. We show that an inclusive $L R H$ is apt. We consider the same population, with normal (no altruism) LRH of $(1,1)$. Let an altruistic act leave the donor, $\mathrm{D}$, with the loss of its first offspring ( $\operatorname{cost} c$ ) and an LRH of $(0,1)$, yielding the direct fitness numbers $S_{3}=1, \lambda_{3}=1$, $T_{3}=2.00$, and $B_{3}=34$ (the following generations are supposed to reproduce normally). The latter number compares to the non-altruistic case by the ratio $B_{3} / B_{0}=34 / 89=0.38$, estimated as $x=\left(\lambda_{3} / \lambda_{0}\right) * * T_{3}$ $=(1 / 1.62)^{2}=0.38$. It applies also to the "unlucky" mate of $\mathrm{D}$, even if the mate may start out, in the first year without offspring, as but a virtual mate. There may generally be more than one mate, but fewer offspring with genes from mates is born, so the loss can be considered as a loss for the community of mates. The usual procedure, in the model of projection matrices, of counting only the females seems not advisable for altruistic acts where a systematic genetic difference between altruist and mate(s) is well possible.

Let the recipient $\mathrm{R}$ for whom we assume a relatedness $r=0.5$ receive an additional 3 first-year offspring (benefit $b$ ) and an LRH $(4,1), S_{4}=5$, $\lambda_{4}=4.23, T_{4}=1.12$, and (again with normal reproduction of later generations) $B_{4}=254$. This compares to the non-altruistic case by the ratio $B_{4} / B_{0}=254 / 89=2.85$, estimated as $x=\left(\lambda_{4} / \lambda_{0}\right) * * T_{4}=(4.23 /$ 1.62 ) $* * 1.12=2.93$, and applies also to the "lucky" mate of $\mathrm{R}$ (or the lucky community of mates). 
However, as far as the donor's altruistic genotype is concerned, $\mathrm{R}$ counts but one-half, with LRH $(2,0.5)$. Considering first both $\mathrm{D}$ and $\mathrm{R}$ together, they count as 1.5 individuals with a ten-year offspring production of $2,3.5,5.5,9,14.5,23.5,38,61.5,99.5$, and $B_{5}=161$, or additional 27.5 ( $=$ a factor of 1.21) compared to the expected $89 * 1.5=133.5$ without altruistic act. The shared inclusive LRH or combined LRH $(2,1.5)$ for 1.5 individuals, or the average combined $L R H(4 / 3,1)$ per individual, gives $S_{5}=3.5 / 1.5=2.33$ per individual, $\lambda_{5}=1.87, T_{5}=1.35$. Our estimate yields $x=\left(\lambda_{5} / \lambda_{0}\right) * * T_{5}=(1.87 /$ $1.62) * * 1.35=1.21$.

The inclusive LRH of the donor alone is found by putting the recipient's relevant surplus offspring $1 / 2\{(4,1)-(1,1)\}=(1.5,0)$ completely on the donor's account to yield $(1.5,1), S_{6}=2.5, \lambda_{6}=2.00$, $T_{6}=1.32$. The inclusive offspring of $\mathrm{D}$ (i.e., total of both minus "normal" offspring of R) after ten years is $B_{6}=161-89 / 2=116.5$, a factor of 1.31 over the non-altruistic expectation of 89 . Our estimate renders $x=\left(\lambda_{6} / \lambda_{0}\right) * * T_{6}=1.32$.

On the other hand, putting the donor's loss $(1,0)$ completely on the recipient's account yields an inclusive LRH for the altruistic genotype in $\mathrm{R}$ of $(1,0.5)$ for the half-counting individual $\mathrm{R}$, or $(2,1)$ per individual, giving $S_{7}=3, \lambda_{7}=2.41, T_{7}=1.25$. The ten-year output (sum of $\mathrm{D}+\mathrm{R}$ minus "normal" D) is $161-89=72$, to be compared to the nonaltruistic case for $\mathrm{R}$ with output $1 / 2 * 89=44.5$. The ratio is $72 /$ $44.5=1.62$. Our estimate renders $x=\left(\lambda_{7} / \lambda_{0}\right) * * T_{7}=1.64$.

As seen from the standpoint of the recipient, its direct LRH is reduced by the cost of $\mathrm{D}$ who counts $1 / 2$ as seen from R. The inclusive LRH and inclusive fitness of R as primary individual is thus $(3.5,1), S_{8}=4.5$, $\lambda_{8}=3.77, T_{8}=1.13, B_{8}=226.5$. The gain is $B_{8} / B_{0}=226.5 / 89=2.54$, estimated as $\left(\lambda_{8} / \lambda_{0}\right) * * \mathrm{~T}_{8}=2.60$.

The example was chosen such that HAMILTON's [2] famous rule is positively fulfilled, with HAMILTON's number $\mathbf{H}=b r-c>0$. The rule can be directly applied only because cost and benefit occur in the same year or breeding season. In general, a translation into our notation has to be used. It is, obviously,

$$
\mathbf{H} \rightarrow \lambda_{5} / \lambda_{0}>1
$$

(alternatively, $\lambda_{6} / \lambda_{0}>1$ or $\lambda_{7} / \lambda_{0}>1$ ). We illustrate this by a slight change of the conditions: Let the donor $\mathrm{D}$ remain unchanged, with $\mathrm{LRH}=(0,1)$ and $c=1$. However, the recipient $\mathrm{R}$ receives the same additional offspring $b=3$ not in the first, as above, but only in the second year, with $\mathrm{LRH}=(1,4)$. The shared $\mathrm{LRH}$ is $(0.5,3)$ for 1.5 
individuals, yielding $S_{5}=2.33$ (per individual), $\lambda_{5}=1.59, T_{5}=1.82$. HAMILTON's translated rule is not fulfilled. The direct ten-year result $\mathrm{D}+\mathrm{R}$ (if all offspring has normal LRH of $(1,1)$ ) is $B_{5}=129.5$, a slight loss of 4 as compared to the non-altruistic case. The multiplicative loss of $129.5 / 133.5=0.97$ is estimated as $\left(\lambda_{5} / \lambda_{0}\right) * * T_{5}=0.97$. Correspondingly, the inclusive LRH of the donor $(0,2.5)$ gives $S_{6}=2.5$, $\lambda_{6}=1.58, T_{6}=2.00, B_{6}=85, B_{6} / B_{0}=85 / 89=0.95,\left(\lambda_{6} / \lambda_{0}\right) * * T_{6}=$ 0.95 while the inclusive LRH of the half-counting recipient $(0.5,2)+(-1,0)$ gives $(-1,4)$ as effective LRH per one individual with $\quad S_{7}=3, \quad \lambda_{7}=1.56, \quad T_{7}=2.47, \quad B_{7}=40.5, \quad 2 B_{7} / B_{0}=40.5 /$ $44.5=0.91,\left(\lambda_{7} / \lambda_{0}\right) * * T_{7}=0.91$.

The 3 cases (LRH shared D $+\mathrm{R}$, inclusive $\mathrm{D}$, inclusive R) are, of course, equivalent, the loss of 4 in the ten-year total result $B_{5}$ amounting to about $3 \%$ loss for 1.5 individuals $(\mathrm{D}+\mathrm{R}), 5 \%$ for 1 (D), and $9 \%$ for 0.5 individuals $(\mathrm{R})$. In the foregoing example, the additional 27.5 offspring constitute a gain of $21 \%(31 \%, 62 \%)$ over the normal offspring for $1.5(1,0.5)$ individuals.

\section{Conclusions}

As far as the original and primary meaning of the fitness concept is concerned, viz., to describe quantitatively the influence of the reproduction of one individual on the gene distribution in later generations, the present definitions of fitness as one-number quantities are, generally speaking, inadequate. Even the use of two numbers, individual fitness and generation length, can fully account only for the simplest once-alife case of reproduction, but it can provide at least approximate answers to questions that were hitherto unanswerable, especially the primary question of fitness theory as mentioned above. A definite advantage over the use of the growth rate fitness alone is that instead of heavily weighting the offspring by time of birth (a possible problem as discussed by, e.g., BROMMER et al. [1]) a common medium weight is given to the entire offspring. On the other hand, such medium weight is also an advantage over the simple LRS fitness definition that includes no weighting of offspring at all.

Our approach can handle not only cases such as one different LRH so to speak a lucky or, as well, unlucky breeder -, but also successive different LRHs describing, e.g., a heritable reproduction pattern that is displayed by only part of the offspring. Altruistic acts can be included by defining inclusive fitness as the fitness following from an inclusive $L R H$ where the LRHs of different animals are combined. We do not 
claim that our approach to inclusive fitness is new but we did not find it in the literature.

In order to transfer $x$ into further generations, good or bad luck need not be continued. On the contrary, any continuation must be treated as multiplication of generations, or multiplication of different generations' values of $x$.

It should be stressed that our approach can do no more than compare the effects of two different LRHs, i.e., we can compare only the outcome in later generations of two individuals or two sets of individuals. We cannot say anything about the second main question of the fitness concept, namely the effect of a certain LRH on the relative abundance of certain genotypes ("spreading of genes", evolutionary effect). This question cannot be answered without additional information on how often different LRHs occur, and how these are related to certain genes or gene combinations.

Most observed LRHs, as well as most of our examples, would indicate a rapidly growing population. It is, therefore, useful to mention that our results can survive the necessary occasional or - with quantitatively different outcome - quasi-continuous reduction to more or less constant population. One should thus distinguish clearly between the different cases of unlimited growth for several generations (niche filling) with later cessation of growth (full niche) or reduction to a former level (niche emptying, decline of population) on the one hand and, on the other hand, essentially-zero growth of levelled populations living in equilibrium with the environment. If the LRHs are not properly reduced to offspring numbers that actually contribute to the next generation's reproduction, the resulting fitness numbers do not give reliable results. In other words: unreduced observed LRHs and the corresponding fitness numbers are nice for qualitative estimates and comparisons (e.g., the advantage of early births in growing and late births in waning populations) but the reduction to successful LRHs is mandatory for quantitative answers to the first question of fitness theory.

Of course, some kind of repetition of luck is necessary for evolutionary consequences. If luck is somehow genetically fixed, for instance if external circumstances favour the occasional luck of a certain allele, one can, in principle, define an "average hereditary luck" with corresponding average $S, \lambda, T$, and $x$ valid for all animals carrying that particular allele. This would lead back to the usually considered case, mentioned above, where a definition of a generation length is no longer necessary because all averaged animals are assumed to have the same fully hereditary average LRH - and where it is the observer who is left with the problem of obtaining proper data. 
If, on the other hand, no allele, or allele combination, is favoured, any of them will have a chance to be occasionally lucky or unlucky in one particular individual, which thus distinguishes itself from others by any $x \neq 1$ and approximately equalling the $x$ of another lucky/unlucky breeder of perhaps many generations before. Then, as a final result, the allele distribution is not much changed and its multiplicity conserved even if in each single generation the dice seem to favour one particular allele.

\section{Acknowledgement}

There are several people to thank but a special mention goes to BARBARA TONKINLEYHAUSEN for commenting on the manuscript and correcting our English. Particular thanks are due to $\mathrm{H}$. Winkler of the Konrad-Lorenz-Institut für Vergleichende Verhaltensforschung who was very helpful in contributing to the easier understanding of our use of the fitness concept.

\section{References}

[1] Brommer, J. E., Merilä, J., KoKko, H. (2002) Reproductive timing and individual fitness. Ecol. Lett. 5: 802-810

[2] Hamilton, W. D. (1964) The genetic evolution of social behaviour. J. Theor. Biol. 7: 1-52

[3] Hulley, P. E., Pfleiderer, M. (1988) The Coleoptera in poultry manure potential predators on house flies, Musca domestica L. (Diptera, Muscidae). J. Entomol. Soc. South Afr. 51: 17-29

[4] Leyhausen, P. (1993) Social behaviour, cultural development and population density, part 2. Soc. Bio. Hum. Aff. 58(2): 1-12

[5] MCGraW, J. B., CASWELl, H. (1996) Estimation of individual fitness from lifehistory data. Am. Nat. 147: 47-64

[6] WILson, E. O. (1975) Sociobiology. Cambridge, MA: Belknap

Authors' addresses: Prof. Dr. Jörg Pfleiderer, Dr. Mircea Pfleiderer, Institut für Astro- und Teilchenphysik der Leopold-Franzens-Universität Innsbruck, Technikerstraße 25, 6020 Innsbruck, Österreich; Karoo Cat Research, Fish River ZA-5883, South Africa. E-Mail: felis@isat.co.za; joerg.pfleiderer@uibk.ac.at. 